\title{
Multiple angiokeratomas of fordyce
}

\section{Kaoutar Laamari*, Hanane Baybay, Chaymae jroundi, Zakia Douhi, Sara Elloudi and Fatima Zahra Mernissi}

Department of Dermatology Venerology, FEZ, Morocco

\section{Introduction}

Angiokeratomas are rare benign vascular lesions characterized by dilated thin-walled blood vessels lying in the upper part of the dermis, mostly associated with an epidermal reaction such as acanthosis and/or hyperkeratosis. Angiokeratomas of Fordyce are predominantly located on the scrotum and are only rarely found on the penis. We report two cases of angiokeratoma of Fordyce located on the glans penis.

\section{Case report 1}

A 24-year-old healthy male patient presented with multiple, tiny, lesions on the corona of the glans penis since four year. They were soft to firm, non-tender and measuring 2-3 mm in diameter (Figure 1). No Sexual contact was noted. There was no inguinal lymphadenopathy. The dermoscopy showed us some dark lacunae and a white veil. The diagnosis of angiokeratoma was certain for his data (Figure 2).

Routine laboratory investigations were normal and venereal Disease Research Laboratory (VDRL) and human immunodeficiency virus (HIV) tests were negative. Unfortunately, the patient refused a skin biopsy and is lost sight of this day.

\section{Case report 2}

A 54-year-old male patient presented with multiple, tiny, erythematous lesions on the corona of the glans penis since four year. He was treated first as a condyloma causing him a family conflict but without improvement.

Clinical examination showed several well-limited, nonpainful,erythematous papules on the glans penis (Figure 1). Dermoscopy was a very useful tool, and showed some dark-blue lacunae and a white veil (Figure 2).

We completed our investigation with a skin biopsy showing a lining of stratified squamous epithelium, which showed hyperkeratosis and irregular acanthosis. The papillary dermis revealed multiple thin-
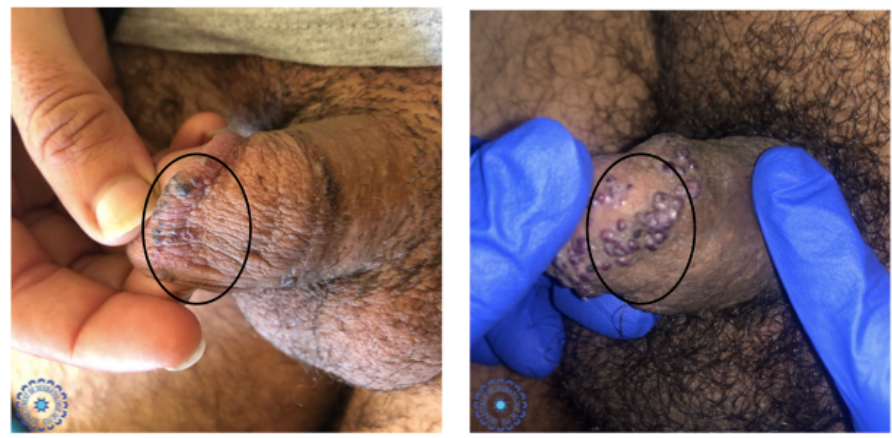

Figure 1. Several well-limited, non-painful, erythematous papules on the glans penis
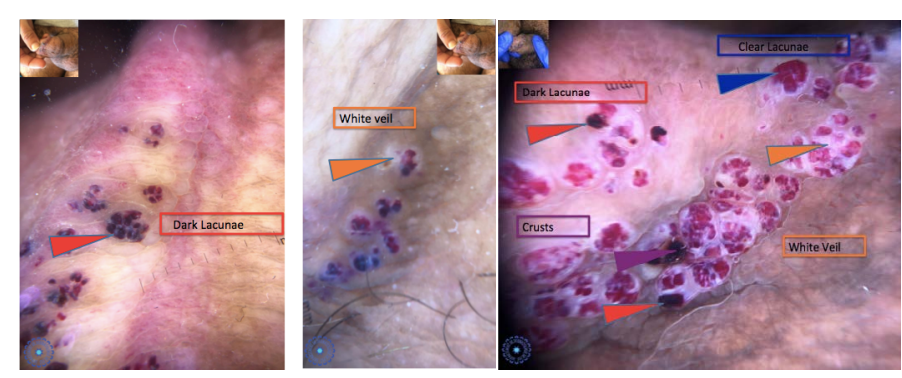

Figure 2. Dermoscopy
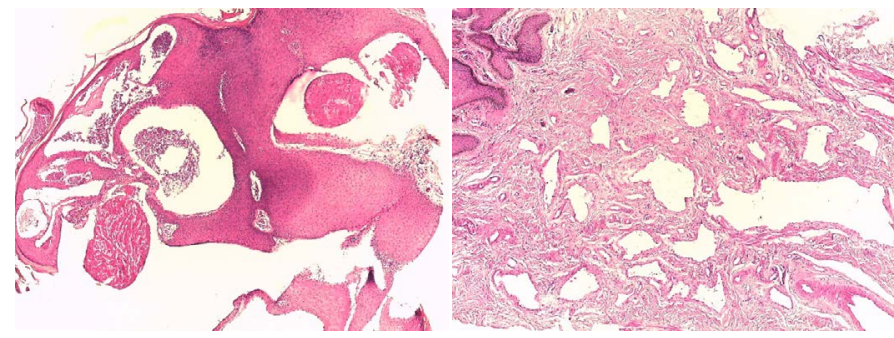

Figure 3. HES staining G x 50 -> Acanthosis papillomatosis + proliferation of dilated vessels between the epidermal ridges; HES staining G x 100 -> Dilated vessels harboring granulomas or thrombosed

walled, ectatic blood vessels containing red blood cells (Figure 3). Laser ablation was the treatment for theses lesions with a good result.

\section{Discussion}

Genital angiokeratomas are benign, usually idiopathic, vascular lesions occurring most commonly on the scrotum or vulva, angiokeratomas of fordyce occurring in glans penis are rare with only few reported cases $[1,2]$.

Pathogenesis of angiokeratomas is unclear but reported to be associated with localized venous hypertension or degeneration of vascular elastic tissue [3].

Angiokeratomas present as red, purple, blue and/or black, typically dome-shaped papules. They range from 0.5 to 5 millimeters in diameter. Five subtypes of angiokeratomas have been described: angiokeratoma

${ }^{\star}$ Correspondence to: Kaoutar Laamari, Department of Dermatology Venerology, FEZ, Morocco, University Hospital Hassan II, Morocco, Email : Kaoutar. laamari1@gmail.com

Received: February 25, 2020; Accepted: April 03, 2020; Published: April 07, 2020 
circumscriptum, angiokeratoma of Fordyce, angiokeratoma of Mibelli, solitary angiokeratoma, and angiokeratoma corporis diffusum [4].

Dermoscopy is a useful tool in differentiating between angiokeratoma and other lesions such as melanoma. Angiokeratoma is characterized by a lacunar or multicomponent pattern, with large, welldelimited, round to oval, red to black areas. There is also a whitish veil attributed to the acanthotic and hyperkeratotic epidermis [5].

The morphologic similarity of angiokeratoma of Fordyce with genital warts or even malignant lesions may pose a diagnostic problem. Dermoscopy and histopathological examination are essential to help for diagnosis [6].

The microscopic finding can show hyperkeratosis and variable degree of acanthosis. The papillary dermis reveals numerous, dilated and congested thin-walled blood vessels which may occasionally be thrombosed.

Multiple potential treatment modalities are available. The various therapeutic methods include cryotherapy, electrocoagulation, laser, sclerotherapy, and surgical excision [7].

\section{References}

1. Feramisco JD, Fournier JB, Zedek DC, Venna SS (2009) Eruptive angiokeratomas on the glans penis. Dermatol Online $J$ 15: 14

2. Leis-Dosil VM, Alijo-Serrano F, Aviles-Izquierdo JA, Lazaro-Ochaita P, LeconaEcheverria M (2007) Angiokeratoma of the glans penis: Clinical, histopathological and dermoscopic correlation. Dermatol Online J 13: 19.

3. Erkek E, Basar MM, Bagci Y, Karaduman A, Bilen CY, et al. (2005) Fordyce angiokeratoma as clues to local venous hypertension. Arch Dermatol 141: 1325-1326.

4. Kanitakis J, Allombert C, Doebelin B, Deroo-Berger MC, Grande S, et al. (2005) Fucosidosis with angiokeratoma. Immunohistochemical \& electronmicroscopic study of a new case and literature review. J Cutan Pathol 32: 506-511.

5. Sahin M, Türel-Ermertcan A, Oztürkan S, Türkdogan P (2006) Thrombosed solitary angiokeratoma of Mibelli simulating malignant melanoma: the importance of dermoscopy in differential diagnosis. J Eur Acad Dermatol Venereol 20: 102-104.

6. Dudani S, Maskara R (2014) Multiple angiokeratomas of Fordyce: Lesions at an unusual site causing a diagnostic dilemma. Indian J Dermatopathol Diagn Dermatol 1: $46-48$

7. Burnett CT, Kouba DJ (2012) A rare case of congenital angiokeratoma of the glans penis treated using a 595-nm pulsed dye laser. Dermatol Surg 38: 2028-2030.

Copyright: $\odot 2020$ Laamari K. This is an open-access article distributed under the terms of the Creative Commons Attribution License, which permits unrestricted use, distribution, and reproduction in any medium, provided the original author and source are credited. 